\title{
Caracterización del estilo de comportamiento basado en infracciones de los conductores de servicio público de Villavicencio
}

\author{
Style Characterization behavior based on breaches \\ of the public service drivers of Villavicencio
}

\section{Caracterização comportamento estilo baseado em violações dos conductores serviço público de Villavicencio.}

\author{
Hernando Castro-Garzón ${ }^{1}$, Lilia Suarez-Puerto ${ }^{2}$
}

\footnotetext{
1 Administrador de empresas agroindustriales, Msc, profesor programa de administración de empresas;

2 Mercadotecnista Agroindustrial, Msc, profesora programa de Mercadeo; grupo de estudio Kavala Universidad de los Llanos; Villavicencio, Colombia.

Email: hcastro@unillanos.edu.co
}

Recibido: 23 de octubre de 2015

Aceptado: 12 de septiembre de 2017

\begin{abstract}
Resumen
Se recopiló y analizó la información obtenida de las 18 empresas transportadoras de servicio público del municipio de Villavicencio. Se realizó un tamizaje inicial con el criterio de representatividad frente al número de vehículos matriculados y las empresas con menos de diez comparendos, de las que se excluyeron diez de ellas; es decir se estudiaron 8 empresas de transporte público. Se clasificaron por el número de infracciones cometidas y el número de vehículos afiliados en función del estilo de comportamiento, clase de vehículo, tipos de infracciones y la frecuencia de las mismas por empresa. El objetivo del estudio fue caracterizar el estilo de comportamiento de los conductores de servicio público a partir de las contravenciones infringidas y poder asociarlos con los problemas de movilidad. Los resultados encontrados determinaron que son 6 las infracciones en las cuales los conductores son recurrentes; permitió identificar que en las empresas observadas el no uso del cinturón de seguridad es la infracción más reiterada, con una participación del $20 \%$, seguido por conducir realizando maniobras altamente peligrosas y usar sistemas móviles de comunicación con un 18\% cada una, luego se encontró que bloquear una calzada o intersección, dejar o recoger pasajeros en sitios distintos a los demarcados y no detenerse ante una luz roja o amarilla o señal de pare tienen respectivamente un 16, 15, y $14 \%$, además hay que mencionar, que la empresa con mayor número de infracciones es Taxi estrella con 225, seguido por la cooperativa de transportadores con 79, luego Transcelutaxi y Rápido los centauros con 75 y 71, además la Nueva urbana de los llanos con 60, Transportes Arimena con 57 y finalmente Transasotax y Taxmeta con 43 y 41 respectivamente. Finalmente se determinó que en promedio el 52,7\% de los conductores analizados poseen un estilo de comportamiento tipo riesgoso y peligroso $(\mathrm{N})$.
\end{abstract}

Palabras clave: Conductor, estilo de comportamiento, movilidad, urbano. 


\begin{abstract}
It was collected and analyzed information from 18 carriers utilities the municipality of Villavicencio. An initial screening was conducted with the criterion of representativeness against the number of registered vehicles and businesses with less than ten infractions, of which ten of them were excluded; public transport 8 companies were studied. They were classified by the number of offenses committed and the number of affiliated vehicles depending on the style of behavior, vehicle class, type of infringements and the frequency of them by now. The aim of the study was to characterize the style of behavior of drivers of public service from the infringed violations and to associate them with mobility problems. The results found that are 6 violations in which drivers are recurring; identified that companies observed the non-use of seat belts is the most repeated infringements, with a share of $20 \%$, followed by drive performing highly dangerous maneuvers and use mobile communication systems with 18\% each, then found that blocking a driveway or intersection, leave or pick up passengers at sites other than demarcated and failing to stop at a red light or stop sign yellow or have respectively 16,15 and $14 \%$, we must also mention that the company with the highest number of infringements is Taxi estrella with 225 , followed by the Cooperativa de Transportadores 79, then the Transcelutaxi and Rápido los centauros with 75 and 71 , plus the Nueva urbana de los llanos 60 , Transportes Arimena 57 and finally Transasotax and Taxmeta with 43 and 41 respectively. Finally it was determined that on average $52.7 \%$ of tested drivers have a style like behavior risky and dangerous $(\mathrm{N})$.
\end{abstract}

Keywords: Driver, mobility, behavioral style, urban.

\title{
Resumo
}

Ele foi recolhido e informações a partir de 18 transportadores utilitários analisados do município de Villavicencio. Uma triagem inicial foi realizado com o critério de representatividade em relação ao número de veículos registrados e empresas com menos de dez intimações, dos quais dez deles foram excluídos; ou seja, os transportes públicos foram estudados 8 empresas. Eles foram classificados pelo número de infracções cometidas e do número de veículos afiliadas, dependendo do estilo de comportamento, classe de veículo, tipo de infracções, bem como a frequência deles até agora. O objetivo do estudo foi caracterizar o estilo de comportamento dos motoristas de serviço público das violações violado e associálos com problemas de mobilidade Os resultados encontrados que são 6 infracções para as quais são recorrentes; Ele identificou que as empresas observaram a não utilização do cinto de segurança são as infracções mais utilizadas , com uma quota de $20 \%$, seguido por unidade realizando manobras altamente perigosas e usar sistemas de comunicações móveis , com 18\% cada, em seguida, encontrou que o bloqueio de uma entrada de automóveis ou cruzamento, deixar ou pegar passageiros em outros locais do que demarcada e des respeito de um sinal vermelho ou amarelo ou sinal pare têm, respectivamente, 16, 15 e 14\%, também devemos mencionar que a empresa com o maior número de infracções é Taxi estrella com 225, seguido pelo Cooperativa de Transportadores 79, em seguida, Transcelutaxi e 75 e Rapido los centauros 71, também Nueva urbana de los llanos 60, Transportes Arimena 57 e, finalmente, Transasotax e Taxmeta com 43 e 41 respectivamente. Finalmente, foi determinado que, em média, 52,7 \% dos condutores testados têm um estilo como comportamento arriscado e perigoso $(\mathrm{N})$.

Palavras-chave: Conductor, estilo de comportamento, a mobilidade, urbano.

\section{Introducción}

Cuando un centro urbano crece de manera acelerada la movilidad generalmente decrece; siendo la confluencia de diferentes actores los que determinan el aumento o la disminución de la misma. Se observa dos componentes cruciales en el uso de vehículos motorizados, los privados y los de uso público, siendo los últimos los de mayor permanencia en la malla vial, por tanto, la relevancia de los conductores de este tipo de vehículos en la definición de la movilidad de una ciudad.

El propósito es el de visualizar un gremio que es crítico en el entramado de la movilidad de una ciudad; para Chaparro y Guerrero (1999). "En Europa y Colombia se considera al gremio de los conductores de camiones como un grupo de alto riesgo"; en efecto es clara la necesidad de considerarlos como sujeto de análisis ya que soportan la responsabilidad de transportar al conglomerado ciudadano.

Considerando que mundialmente es preponderante el logro de estándares seguros para los conductores, y una ruta hacia la movilidad adecuada; la Dirección General de tráfico (2013) sostiene que:

Seguridad vial y movilidad son cuestiones sociales de primer orden por su incidencia sobre la salud de los ciudadanos y sobre su calidad de vida: más de 2.000 personas perdieron la vida en accidentes de tráfico ocurridos en las vías públicas de nuestro país durante el año 2011. Pero mas allá de las consecuencias sobre la mortalidad, las lesiones por tráfico impactan en la calidad de vida de los individuos y modifican sus relaciones sociales, laborales y familiares, como consecuencia de las lesiones graves no mortales.

Como se afirma anteriormente el resultado de una adecuada gestión en cuanto a preparación y concientiza- 
ción de los conductores de servicio público mejoraría ostensiblemente tanto la movilidad como el bienestar de las personas.

Para comprender mejor la importancia del papel del transporte público como elemento fundamental para un proceso de sostenibilidad de la movilidad, que aún en Villavicencio no se ha iniciado, es necesario ver la función clave de los conductores en el sistema de transporte público, ya que son ellos quienes a diario soportan la responsabilidad de movilizar a cientos de personas en sus vehículos, sin embargo su comportamiento habitual puede incidir en la congestión de carreteras urbanas.

En efecto, la consideración de la complejidad y la permanente interacción de imbricadas relaciones de diverso orden, generan que el papel de los conductores sea vital para la definición de una ciudad sostenible para Rodrigue (2013) "conceptualmente, el sistema de transporte urbano es un intrincado vínculo con la forma y la estructura espacial urbana", donde denota el papel vinculante del conductor como accionante en la generación de movilidad urbana.

Prosiguiendo esta misma línea de la movilidad y cultura ciudadana Ballén (2007) presenta la importancia que se le ha otorgado a las vías, a los medios de transporte, además de su impacto al paisaje y a la calidad de vida en la ciudad; señala que el derecho a la movilidad debe ser vinculante con lo justo, lo correcto, lo legítimo, con la aproximación a la igualdad real, con productividad, rendimiento económico, integración territorial, espacio público y medio ambiente.

A continuación, se muestra la utilidad que genera una encuesta origen destino para la ciudad de Bogotá en términos de movilidad:

La encuesta de movilidad -dice el autor del estudio- es el análisis e investigaciones de los comportamientos sociales asociados a la movilidad: motivación, comportamiento, usos del tiempo y opinión. La encuesta también recoge una dimensión más subjetiva, en la que se quiere conocer las percepciones, las predisposiciones y las valoraciones de los ciudadanos hacia la movilidad Martin (2011).

Como se afirma arriba, se buscaba interactuar con el ciudadano en condiciones que permitan conocer la dinámica de sus desplazamientos por la ciudad. Los resultados son útiles para la toma de decisiones en la formulación de los planes de movilidad y en términos generales puede afirmarse que las encuestas origen destino, con las características de modos de transporte y sus frecuencias, son esenciales para la determinación de acciones frente a la movilidad.

En consonancia, una investigación sobre los patrones de comportamiento de los conductores de transmilenio y los aspectos que influyen en ellos, González (2012) concluye:

No todos los conductores de Transmilenio tienen adecuados patrones de comportamiento en contextos de tráfico. El análisis cualitativo del trabajo de campo realizado evidencia que en estos operarios predominan con una participación del $60 \%$ comportamientos de tipo $\mathrm{N}$ es decir conductas peligrosas, arriesgadas, aceleradas lo cual genera un alto impacto en la movilidad.

Considerar ahora, el papel del conductor es fundamental en el logro de una movilidad adecuada; por eso se analiza la injerencia que tiene la formación de futuros conductores ya que la influencia del ejemplo de los padres en el estilo de conducción de sus hijos determinará el accionar del futuro conductor; sobre este tópico expresa Montoro (2013):

Los jóvenes entran en una etapa del ciclo vital en la que su propia personalidad, y en muchas ocasiones la sociedad y el entorno les potencia, refuerza y fomenta valores que dentro de los vehículos con motor son un serio peligro para la movilidad segura, como la agresividad, la competitividad, el exhibicionismo, la búsqueda de emociones o la autoafirmación y sobre-valoración de sus capacidades en el manejo de las máquinas.

Habría que decir también, que la conducta es esencial para lograr un adecuado comportamiento en la vía, siendo garante para el ejercicio de una adecuada movilidad.

Estas observaciones se relacionan también con las respuestas actitudinales; puesto que conductores que se encuentran con diversas contravenciones de la normatividad su distintivo actitudinal es negativo (Rodríguez, 2009), estableciendo un rechazo hacia los demás actores de la movilidad reflejado en el comportamiento hacia ellos (Montoro et ál., 2001). Por otro lado, Yagil (2001) determinó que el género masculino adopta el hecho de conducir, como vehículo de poder, o para alcanzar elementos incitadores de adrenalina, generando conductas agresivas frente a los comportamientos también agresivos de otros ciudadanos, aclarando que esta conducta también se refleja en las mujeres en una menor escala. Se debe agregar que, para Li, et al.,(2008) estar emocionalmente afectado, como por ejemplo con abatimiento, se convierte en una herramienta catalizadora entre la personalidad, conductas y actitudes proclives al riesgo al volante; en el análisis determinaron que un nivel alto de abatimiento no se ve 
reflejado en respuestas de alto riesgo en los conductores. Todavía cabe señalar, que la hostilidad no actuaba como determinante de la personalidad, en cambio la percepción sobre el entorno era lo que establecía una respuesta conductual riesgosa (Renge, 2008).

Prosiguiendo el análisis, se detectó que los conductores jóvenes tienen mayores índices de accidentalidad, "La relación existente entre los jóvenes y los accidentes de tráfico está condicionada por un conjunto de factores de carácter psicológico y sociocultural, que en algunos casos, es percibida como una conjunción inevitable" (Race, 2012). Como resultado, los factores comportamentales y de orden sicológico influyen en las reacciones en la vía y finalmente en la movilidad.

Definitivamente, son los comportamientos los que definen una adecuada movilidad, expresado por (Parrado, 2001) en los siguientes términos; "debe haber cambio en la mentalidad de los usuarios de los distintos modos de transporte, hasta una actuación muy concreta en el parque automotor usuario de la infraestructura"; esto es necesario para iniciar un proceso de mejoramiento de la movilidad de cualquier ciudad.

Como se afirmó arriba, es necesario un proceso de concientización de los usuarios y los conductores, que están expuestos a muchos condicionamientos como lo expresa (Crawford, 2005):

Los cambios obedecen a una multitud de influencias, de las cuales las más importantes son: Los valores, la filosofía, los sistemas de gobierno, el tamaño de la población, la sensibilidad artística, las técnicas de diseño, los métodos de construcción, las técnicas de pavimento y la tecnología de transporte.

Lo anterior quiere decir, que es necesario generar un escenario adecuado con los actores pertinentes para inducirlos a comportamientos tendientes a establecer una movilidad eficiente con miras a iniciar el camino a la sostenibilidad.

A continuación, se considera un accionar tendiente al mejoramiento de la movilidad, por ende del bienestar ciudadano, como un fin de la municipalidad reduciendo las condiciones negativas en la población (OMLT, 2012). Dicho lo anterior, ciudad sostenible es aquella que reconoce el actuar de sus ciudadanos con procesos adecuados de intermodalidad y desestímulo al uso del vehículo privado (Comisión de las Comunidades Europeas, 2012). Ahora, se puede decir, que la movilidad es un problema propio de las sociedad contemporánea y sobre su funcionamiento hay abundantes ejemplos alrededor del mundo (Movilia, 2008). Dicho lo anterior, se entiende que la movilidad es un asun- to que compete a toda la ciudadanía; sin importar su papel como agente o actor en la misma; en consonancia para lograr un equilibrio entre las necesidades de movilidad y accesibilidad (Obra caja Social Madrid, 2008). De ahí que, la movilidad en via hacia la sostenibilidad es el proyecto más ambicioso de las urbes en la actualidad.

Para concluir, se observa que para la transformación a través de la pedagogía, la formación y el conocimiento, es importante la participación de los actores sociales en especial conductores y peatones (Sierra, 2004).

En contraste con todo lo anterior, el objetivo del presente estudio fue caracterizar el estilo de comportamiento de los conductores de servicio público a partir de las contravenciones infringidas y poder asociarlos con los problemas de movilidad que se suscitan en la ciudad de Villavicencio.

\section{Materiales y métodos}

La metodología empleada en la investigación corresponde al enfoque cuantitativo, a partir de los datos recopilados de los comparendos impuestos por la Secretaría Municipal de Movilidad de Villavicencio (2013) del año 2012; se procedió a seleccionar las empresas que fueran representativas para el estudio, es decir, aquellas que contaran con un número significativo de infracciones de tránsito. De esta manera de las 18 empresas de servicio público existentes en la ciudad de Villavicencio, el objeto de estudio se centró en 8 de ellas, para un total de 1.497 infracciones de tránsito. Para el estudio se contemplaron empresas de transporte público colectivo (TPC) y de transporte público individual (taxis).

Para el análisis de los datos, se emplearon herramientas de estadística descriptiva como medidas de tendencia central y de dispersión, para determinar el comportamiento de los datos. Los comparendos fueron clasificados por empresas para calcular aquellas que presentan mayor número de faltas. También se realizó una categorización por tipo de vehículo para determinar aquellos medios de transporte más riesgosos en la ciudad. Finalmente se tuvo en cuenta el tamaño del parque automotor para calcular el número de comparendos pero en relación a los autos asociados a la empresa.

Con los datos fue posible caracterizar las diferentes faltas cometidas por los conductores. Esta tipología permitió conocer las infracciones más frecuentes por los conductores y en las empresas. La información consolidada a partir de los comparendos permite caracterizar las conductas de los prestadores de servicio 
público en la ciudad. La cantidad y tipo de infracciones incurridas por los conductores, dan cuenta de las acciones peligrosas cometidas por los prestadores de servicio público, relacionándola con un factor de comportamiento establecido por Fernandez (1998) que determina los estilos de los conductores en tipo $\mathrm{S}$ y $\mathrm{N}$ que definen dos patrones de personalidad uno con prevalencia de reacciones seguras, responsabilidad en la conducción, prudencia, el estado ideal del conductor, y el otro con comportamientos al conducir riesgosos y peligrosos. Se observó la frecuencia de infracciones cometidas por conductor, ya que en palabras de Rodríguez (2009), las respuestas actitudinales de los conductores que se encuentran con diversas contravenciones de la normatividad, su distintivo actitudinal es negativo.

\section{Resultados}

Como se puede apreciar en la tabla 1 se analizaron 8 empresas, por su representatividad en el número de infracciones registradas, donde conviene subrayar que la empresa Taxmeta tiene el menor número (90) y la empresa Taxi estrella presenta el número mayor (589) de estas. Es necesario recalcar que la diferencia entre la empresa con mayor número de infracciones y la que le antecede es de (400) infracciones; de donde resulta un número total de 1,497 infracciones entre las empresas analizadas.

Tabla1. Número de infracciones por empresa.

\begin{tabular}{|l|c|}
\hline \multicolumn{1}{|c|}{ Empresas } & \multicolumn{1}{|c|}{$\begin{array}{c}\mathbf{N}^{\circ} \\
\text { infracciones }\end{array}$} \\
\hline Taxmeta & 90 \\
\hline Transasotax & 103 \\
\hline Nueva urbana de los llanos & 108 \\
\hline Transportes Arimena & 128 \\
\hline Cooperativa de transportadores & 135 \\
\hline Rápido los centauros & 155 \\
\hline Transcelutaxi Ltda. & 189 \\
\hline Taxi estrella & 589 \\
\hline Total comparendos & 1497 \\
\hline
\end{tabular}

Elaboración propia, datos secretaría de movilidad Villavicencio 2013.

En la tabla 2 se observa las 6 infracciones de mayor recurrencia cometidas por los conductores de servicio público; el listado inicia con un total de 129 infracciones correspondiente al no uso del cinturón de seguridad y termina con 89 infracciones por no detenerse en una luz roja o amarilla de semáforo o señal de pare. En consecuencia, estas infracciones ascienden a 651 lo cual representa un $43.49 \%$ de los 53 tipos de infracciones cometidas por los conductores analizados.

Tabla 2. Tipo y frecuencia de comparendos.

\begin{tabular}{|l|c|c|}
\hline \multicolumn{1}{|c|}{ Descripción } & Código & $\begin{array}{c}\mathbf{N}^{\circ} \\
\text { Infracciones }\end{array}$ \\
\hline $\begin{array}{l}\text { No utilizar el cinturón de seguridad } \\
\text { por parte de los ocupantes del } \\
\text { vehículo }\end{array}$ & C06 & 129 \\
\hline $\begin{array}{l}\text { Conducir realizando maniobras } \\
\text { altamente peligrosas e irresponsables }\end{array}$ & D07 & 118 \\
\hline $\begin{array}{l}\text { Usar sistemas móviles de } \\
\text { comunicación o teléfonos instalados } \\
\text { en los vehículos al momento de } \\
\text { conducir }\end{array}$ & C38 & 114 \\
\hline $\begin{array}{l}\text { Bloquear una calzada o intersección } \\
\text { con un vehículo }\end{array}$ & C03 & 102 \\
\hline $\begin{array}{l}\text { Dejar o recoger pasajeros en sitios } \\
\text { distintos a los demarcados }\end{array}$ & C19 & 99 \\
\hline $\begin{array}{l}\text { No detenerse ante una luz roja o } \\
\text { amarilla de semáforo, una señal de } \\
\text { "PARE" }\end{array}$ & D04 & 89 \\
\hline TOTAL & 651 \\
\hline
\end{tabular}

Elaboración propia, datos secretaria de movilidad Villavicencio 2013.

La tabla 3 permite identificar que en las empresas observadas de transporte público el no uso del cinturón de seguridad es la infracción mas reiterada, con una participación del $20 \%$, seguido por conducir realizando maniobras altamente peligrosas y usar sistemas móviles de comunicación con un $18 \%$ cada una, luego se encontró que bloquear una calzada o intersección, dejar o recoger pasajeros en sitios distintos a los demarcados y no detenerse ante una luz roja o amarilla o señal de pare tienen respectivamente un 16,15 , y $14 \%$.

Hay que mencionar que la empresa con mayor número de infracciones es Taxi estrella con 225, seguido por la cooperativa de transportadores con 79 , luego Transcelutaxi y Rápido los centauros con 75 y 71, además la Nueva urbana de los llanos con 60, Transportes Arimena con 57 y finalmente Transasotax y Taxmeta con 43 y 41 respectivamente.

Dicho lo anterior, se observó que la variación porcentual entre las infracciones cometidas no sobrepasa el $2 \%$, de donde se infiere que tienen una tendencia a la regularidad. 
Tabla 3. Correlación tipo de infracción- empresa

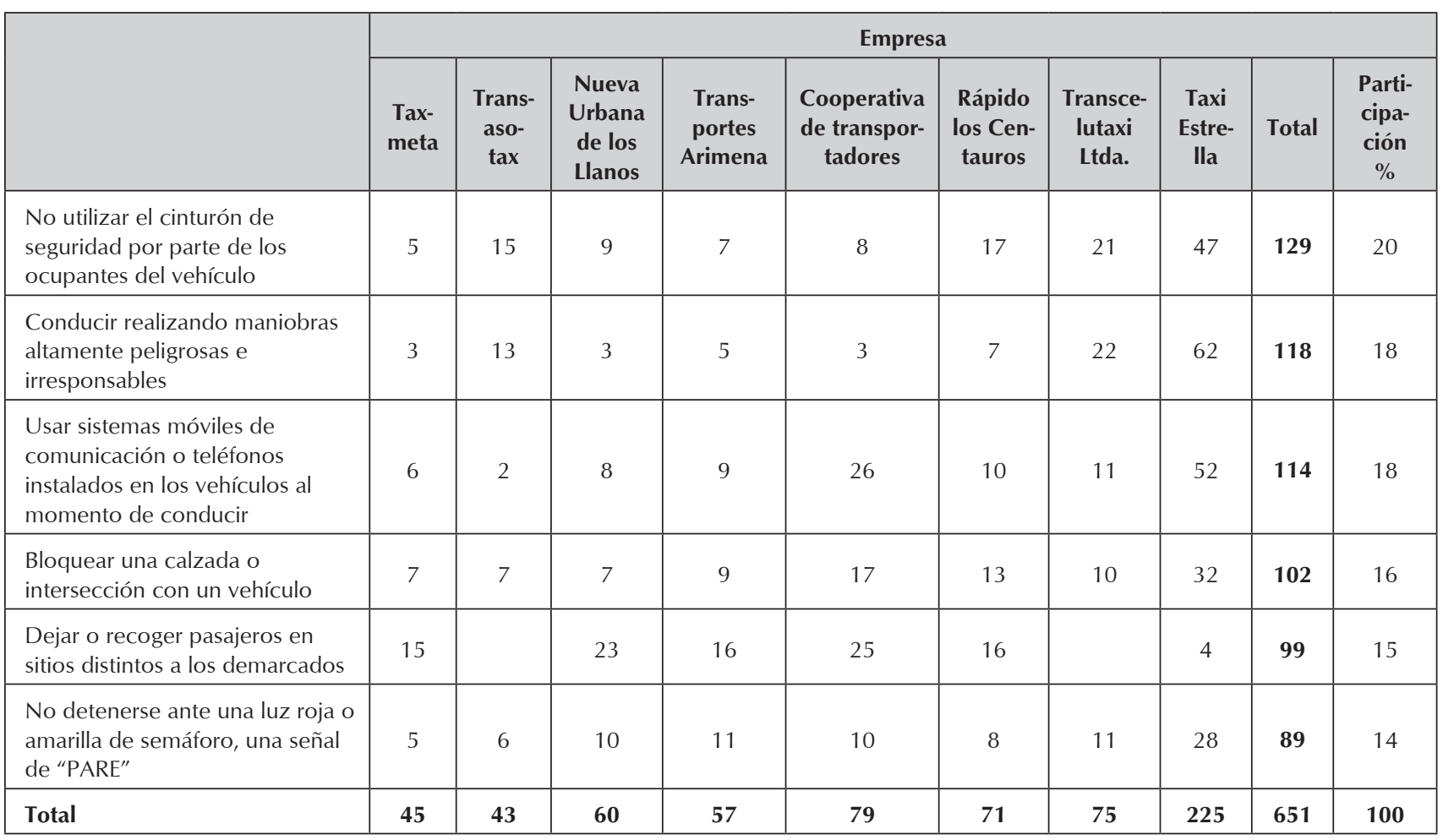

Elaboración propia, datos secretaría de movilidad Villavicencio 2013.

En la tabla 4 se pudo observar que el total de conductores reincidentes son 200; teniendo en cuenta que 164 corresponden a una recurrencia de 2 infracciones por conductor; en donde para 28 conductores se registran 3 infracciones para cada uno, y en el caso de 4 y 5 infracciones el registro es de 6 y 2 conductores respectivamente. Dicho lo anterior, las empresas con conductores con 5 infracciones son Taxi estrella y Taxmeta, con 4 infracciones Nueva urbana de los llanos, Taxi estrella, Taxmeta, y Transcelutaxi Ltda, seguido por 3 y 2 infracciones por conductor con participación de todas las empresas estudiadas.

La tabla 5 mostró que la relación entre número de vehículos afiliados y las infracciones cometidas es inversamente proporcional ya que se observó que Taxmeta posee un número bajo de afiliados pero una proporción alta de infracciones por vehículo 61.2\%; en contraste con Taxi estrella que cuenta con un total de 1,245 vehículos afiliados con una proporción del $47.3 \%$ de infracciones. Habría que decir también que tan solo Transasotax tiene un porcentaje inferior al $45 \%$ de infracciones por vehículo afiliado, hecha esta salvedad, las demás empresas estudiadas presentan proporciones entre el 47.6 y $61.6 \%$ de infracciones por vehículo afiliado, proporcionalmente hablando.

\section{Discusión}

El propósito principal del presente trabajo fue realizar una primera aproximación en la validación de elementos cuantitativos que permitiera un punto de partida para un análisis cualitativo de los comportamientos de los conductores de servicio público de la ciudad de Villavicencio.

Con respecto a la teoría base de este estudio, Fernández (1998) y Rodríguez (2009), se estableció que las respuestas actitudinales de los conductores que se encuentran con diversas contravenciones de la normatividad, su distintivo actitudinal es negativo.

Se debe agregar que, los conductores analizados se pueden agrupar en tipo $\mathrm{N}$ ya que presentan un alto índice de infracciones, además representan un distintivo actitudinal negativo ya que son reincidentes. Así mismo según González (2012) "El análisis cualitativo del trabajo de campo realizado evidencia que en estos operarios (conductores transmilenio) predominan con una participación del $60 \%$ comportamientos de tipo $\mathrm{N}$ es decir conductas peligrosas, arriesgadas, aceleradas lo cual genera un alto impacto en la movilidad"; por consiguiente se estableció que el $52.8 \%$ de los conductores observados están 
Tabla 4. Reincidencia en infracciones por empresa

\begin{tabular}{|l|c|c|c|c|c|}
\hline \multicolumn{1}{|c|}{ Empresa } & 2 infracciones & $\mathbf{3}$ infracciones & $\mathbf{4}$ infracciones & $\mathbf{5}$ infraccion & Total \\
\hline Cooperativa de transportadores & 18 & 1 & & 19 \\
\hline Nueva urbana de los llanos & 10 & 1 & 1 & 12 \\
\hline Rápido los centauros & 14 & 2 & & 16 \\
\hline Taxi estrella & 65 & 13 & 1 & $\mathbf{8 0}$ \\
\hline Tax meta & 9 & 2 & & $\mathbf{1 3}$ \\
\hline Transportes Arimena & 15 & 13 & 2 & & 19 \\
\hline Transasotax & 20 & 3 & 3 & & 15 \\
\hline Transcelutaxi Itda & 164 & 28 & 6 & 26 \\
\hline Total & & & & 200 \\
\hline
\end{tabular}

Elaboración propia, datos secretaría de movilidad Villavicencio 2013.

Tabla 5. Relación total entre infracciones y parque automotor afiliado por empresa.

\begin{tabular}{|l|c|c|c|}
\hline \multicolumn{1}{|c|}{ Empresas } & $\mathbf{n}^{\circ}$ infraccion & Parque automotor & Proporción \\
\hline Transasotax & 103 & 255 & $40,4 \%$ \\
\hline Taxi estrella & 589 & 1245 & $47,3 \%$ \\
\hline Transportes arimena & 128 & 257 & $49,8 \%$ \\
\hline Rapido los centauros & 155 & 303 & $51,2 \%$ \\
\hline Transcelutaxi Itda & 189 & 362 & $52,2 \%$ \\
\hline Nueva urbana de los llanos & 108 & 202 & $53,5 \%$ \\
\hline Taxmeta & 90 & 147 & $61,2 \%$ \\
\hline Cooperativa de transportadores & 135 & 219 & $61,6 \%$ \\
\hline
\end{tabular}

Elaboración propia, datos secretaría de movilidad Villavicencio 2013.

caracterizados en este tipo, en términos cuantitativos.

Conviene subrayar que, según el tipo de infracciones recurrentes, las conductas de los conductores son riesgosas y de tentativa contra los demás usuarios de la movilidad urbana; estableciendo un rechazo hacia los demás actores de la movilidad reflejado en el comportamiento hacia ellos (Montoro et ál., 2001).

Hay que mencionar además, que se asocia teóricamente la variable independiente, infracciones con la hostilidad, ya que se estableció que según Rodríguez (2009) los infractores son proclives a estas conductas y se refleja en los comportamientos de rechazo contra los demás participantes de la movilidad. A demás acentuado por los análisis de Lancaster y Ward (2002), donde se determina que los conductores con incipiente posibilidad de control de la agresividad se ven avocados a contravenir la normatividad.

Los anteriores conceptos determinaron que los conductores objeto de estudio son reconocidos de forma cuantitativa como proclives al riesgo y considerados tipo $\mathrm{N}$ con una tendencia a conductas conflictivas en contra de los demás actores de la movilidad urbana de villavicencio.

Para concluir, se hace necesario ahondar en elementos cualitativos para obtener una mejor comprensión de las respuestas actitudinales de los conductores, ya que como se observó, se presenta también un alto número de conductores con comportamientos adecuados e incluso sin contravenciones; por tanto la interpretación 
cualitativa facilitaría más aún el análisis tendencial de los conductores.

\section{Referencias}

Ballén DF. Estudio derecho a la movilidad. La experiencia de Bogotá D.C. Universidad Militar Nueva Granada. Bogotá, Prolegomenos Derechos Y Valores. 2007;10(20):169-181.

Chaparro NPE, Guerrero J. Condiciones de trabajo y salud en conductores de una empresa de transporte público urbano en Bogotá D.C, Rev. Salud Pública. 1999; 3(2): 171-187.

Comisión de las comunidades europeas. (2007). Hacia una nueva cultura de la movilidad urbana. Bruselas. Pp.1-25.

Crawford JH. (2005). A Brief History of Urban Form. Street Layout Through the Ages, Carfree.com. Pp. 31-54

Fernández SJL \& Departamento I+D (1998). Batería de conductores $(\mathrm{BC}) .\left(3^{\mathrm{a}}\right.$ ed.). Madrid: TEA Ediciones, S.A.

Direccion General de tráfico. Plan de investigación en seguridad vial y movilidad. Madrid 2013. www.dgt.es/es/seguridad-vial/investigacion/planes-investigacion

González JF. 2012. Patrones de comportamiento de los conductores del sistema transmilenio en la ciudad de Bogotá. Universidad Libre.

Lancaster R, Ward R. 2002. The contribution of individual factors to driving behaviour: Implications for managing work-related road safety. Health and Safety Executive, HSE Books.

Li J, Xie X, Hu T. (2008, julio). The effect of mood and emotion on risky driving. Ponencia presentada en el XXIX International Congress of Psychology. Berlín, Alemania.

Martin H. 2011. Segundo encuentro iberoamericano de movilidad sostenible. Bogotá, Universidad Nacional de Colombia.

Montoro GL. 2013. La transmisión de valores para una movilidad segura, Conferencias "Movilidad responsable. Valores en movimiento", realizado por la fundación Abertis, Madrid.
Montoro GL, Alonso F, Esteban C, Toledo F. 2001. Manual de seguridad vial: El factor Humano, Barcelona, Ariel INTRAS.

Movilia. 2008. Encuesta de movilidad Madrid.

Obra Caja Social Madrid. 2012. Movilidad Urbana Sostenible: Un reto energético y ambiental, Madrid.

OMLT. 2012. Observatorio de la Movilidad, Logística y Transporte, Universidad Nacional, Bogotá.

Parrado DCC. 2002. Metodología para la ordenación del territorio bajo el prisma de la sostenibilidad 2001, (Tesis Doctoral), Universitat Politècnica de Catalunya $\iota_{\iota}$ España. http://hdl.handle. net/10803/6984

Race. 2012. Seguridad vial, Informe jóvenes conductores, Madrid.

Renge V. 2008. Combining social axioms with personality measures and self-reported driving behavior in predicting traffic accidents, Ponencia presentada en el XXIX International Congress of Psychology. Berlín, Alemania.

Rodrigue JP, Comtois C, Slack B . 2013. The Geography of Transport Sistems. Third Edition. Routledge. New York.

Rodríguez MA. 2009. Los efectos del control externo e interno en la prevención de la conducción antisocial, Ponencia presentada en el $\mathrm{V}$ Congreso Nacional de Psicología Jurídica y Forense. Granada, España.

Secretaria de movilidad de Villavicencio. Área de sistemas. Enero de 2013.

Sierra SEY. 2004. Una trama particular: pedagogía ciudadana, instituciones, proceso histórico y liderazgo, Análisis en la ciudad de Bogotá del primer periodo Mockus 1995-1997,Bogotá.

Yagil D. Reasoned action and irrational motives: A prediction of drivers' intention to violate traffic laws. J Appl Soc Psychol. 2001;31(4):720-739. 